\title{
Molecular epidemiology of SARS-CoV-2 isolated from COVID-19 family clusters
}

Gunadi $^{{ }^{*}+}$, Hendra Wibawa ${ }^{2+}$, Mohamad Saifudin Hakim ${ }^{3}$, Marcellus ${ }^{4}$, Ika Trisnawati ${ }^{5}$, Riat El Khair ${ }^{6}$, Rina Triasih', Irene $^{8}$, Afiahayati ${ }^{9}$, Kristy Iskandar $^{10}$, Siswanto ${ }^{11}$, Nungki Anggorowati ${ }^{12}$, Edwin Widyanto Daniwijaya ${ }^{13}$, Endah Supriyati ${ }^{14}$, Dwi Aris Agung Nugrahaningsih ${ }^{15}$, Eko Budiono ${ }^{5}$, Heni Retnowulan ${ }^{5}$, Yunika Puspadewi ${ }^{6}$, Ira Puspitawati ${ }^{6}$, Osman Sianipar ${ }^{6}$, Dwiki Afandy ${ }^{4}$, Susan Simanjaya ${ }^{4}$, William Widitjiarso ${ }^{4}$, Dyah Ayu Puspitarani ${ }^{4}$, Fadil Fahri ${ }^{4}$, Untung Riawan ${ }^{4}$, Aditya Rifqi Fauzi ${ }^{4}$, Alvin Santoso Kalim ${ }^{4}$, Nur Rahmi Ananda ${ }^{5}$, Amalia Setyati ${ }^{7}$, Dwikisworo Setyowireni ${ }^{7}$, Ida Safitri Laksanawati ${ }^{7}$, Eggi Arguni ${ }^{7}$, Titik Nuryastuti ${ }^{3}$ and Tri Wibawa ${ }^{3}$ on behalf of the Yogyakarta-Central Java COVID-19 study group

\begin{abstract}
Background: Transmission within families and multiple spike protein mutations have been associated with the rapid transmission of SARS-CoV-2. We aimed to: (1) describe full genome characterization of SARS-CoV-2 and correlate the sequences with epidemiological data within family clusters, and (2) conduct phylogenetic analysis of all samples from Yogyakarta and Central Java, Indonesia and other countries.

Methods: The study involved 17 patients with COVID-19, including two family clusters. We determined the fullgenome sequences of SARS-CoV-2 using the Illumina MiSeq next-generation sequencer. Phylogenetic analysis was performed using a dataset of 142 full-genomes of SARS-CoV-2 from different regions.

Results: Ninety-four SNPs were detected throughout the open reading frame (ORF) of SARS-CoV-2 samples with $58 \%$ (54/94) of the nucleic acid changes resulting in amino acid mutations. About $94 \%(16 / 17)$ of the virus samples showed D614G on spike protein and 56\% of these (9/16) showed other various amino acid mutations on this protein, including L5F, V83L, V213A, W258R, Q677H, and N811I. The virus samples from family cluster-1 $(n=3)$ belong to the same clade $\mathrm{GH}$, in which two were collected from deceased patients, and the other from the survived patient. All samples from this family cluster revealed a combination of spike protein mutations of D614G and V213A. Virus samples from family cluster- $2(n=3)$ also belonged to the clade $\mathrm{GH}$ and showed other spike protein mutations of L5F alongside the D614G mutation.
\end{abstract}

Conclusions: Our study is the first comprehensive report associating the full-genome sequences of SARS-CoV-2 with the epidemiological data within family clusters. Phylogenetic analysis revealed that the three viruses from family cluster-1 formed a monophyletic group, whereas viruses from family cluster-2 formed a polyphyletic group indicating

*Correspondence: drgunadi@ugm.ac.id

${ }^{\dagger}$ Gunadi and Hendra Wibawa contributed equally to this work

${ }^{1}$ Pediatric Surgery Division, Department of Surgery/Genetics Working

Group, Faculty of Medicine, Public Health and Nursing, Universitas

Gadjah Mada/Dr, Sardjito Hospital, Jl. Kesehatan No. 1, Yogyakarta 55281,

Indonesia

Full list of author information is available at the end of the article

(c) The Author(s) 2021. Open Access This article is licensed under a Creative Commons Attribution 4.0 International License, which permits use, sharing, adaptation, distribution and reproduction in any medium or format, as long as you give appropriate credit to the original author(s) and the source, provide a link to the Creative Commons licence, and indicate if changes were made. The images or other third party material in this article are included in the article's Creative Commons licence, unless indicated otherwise in a credit line to the material. If material is not included in the article's Creative Commons licence and your intended use is not permitted by statutory regulation or exceeds the permitted use, you will need to obtain permission directly from the copyright holder. To view a copy of this licence, visit http://creativecommons.org/licenses/by/4.0/. The Creative Commons Public Domain Dedication waiver (http://creativeco $\mathrm{mmons}$.org/publicdomain/zero/1.0/) applies to the data made available in this article, unless otherwise stated in a credit line to the data. 
there is the possibility of different sources of infection. This study highlights how the same spike protein mutations among members of the same family might show different disease outcomes.

Keywords: COVID-19 severity, Family cluster, Multiple spike protein mutations, Phylogenetic analysis, SARS-CoV-2 transmission, Whole genome sequencing

\section{Introduction}

Many countries are still struggling to control the COVID19 pandemic, including Indonesia [1, 2]. On April 15, 2021, Indonesia recorded 1,583,182 confirmed COVID19 cases with 42,906 deaths and infection rate of approximately 6000 cases/day [3].

One of the most important factors affecting the rapid spreading of COVID-19 is transmission within families $[4,5]$. Genomic epidemiology has been suggested to be important to fill the gaps in identifying the SARS-CoV-2 infection sources [6]. However, to our best knowledge, no reports have described the genomic epidemiology within family clusters [6-8]. Moreover, multiple spike protein mutations have been associated with a higher transmissibility of SARS-CoV-2 [9]. In this study, we aimed to: (1) perform full genome characterization of SARS-CoV-2 and correlate the sequences with the epidemiological data within family clusters in Indonesia, and (2) conduct phylogenetic analysis of all samples from Yogyakarta and Central Java, Indonesia, involving the family clusters, and virus data from other regions in Indonesia.

\section{Methods \\ SARS-CoV-2 samples}

We collected all virus samples of confirmed COVID19 patients from Yogyakarta and Central Java provinces from June to November 2020. All nasopharyngeal samples were collected in viral transport media (DNA/RNA Shield ${ }^{\mathrm{TM}}$ Collection Tube with Swab, Zymo Research, CA, United States) and transported to four COVID-19 diagnostic laboratories in Yogyakarta province: (1) Molecular Diagnostic Laboratory, Integrated Laboratory Unit, Dr. Sardjito Hospital; (2) Department of Microbiology and Laboratorium Diagnostik Yayasan Tahija World Mosquito Program, Faculty of Medicine, Public Health and Nursing, Universitas Gadjah Mada; (3) Balai Besar Teknik Kesehatan Lingkungan dan Pengendalian Penyakit (BBTKLPP), Yogyakarta; and (4) Disease Investigation Center, Wates, Yogyakarta. SARS-CoV-2 was detected by RealQ 2019-nCoV Detection Kit (BioSewoom, Seoul, South Korea) with LightCycler ${ }^{\circledR} 480$ Instrument II (Roche Diagnostics, Mannheim, Germany).

\section{Full-genome sequencing}

First, we performed RNA extraction of 19 nasopharyngeal swab samples by a QiAMP Viral RNA mini kit
(Qiagen, Hilden, Germany), synthesized the doublestranded cDNA by Maxima H Minus Double-Stranded cDNA Synthesis (Thermo Fisher Scientific, MA, United States), and purified the cDNA using a GeneJET PCR Purification Kit (Thermo Fisher Scientific, MA, United States). For library preparations, we utilized the Nextera DNA Flex for Enrichment using Respiratory Virus Oligos Panel, whereas for full-genome sequencing, we used next generation sequencing (NGS) applied in the Illumina MiSeq instrument (Illumina, San Diego, CA, United States) with Illumina MiSeq reagents v3 150 cycles $(2 \times 75$ cycles $)$. We excluded two samples for further bioinformatics analysis because of low coverages. Our sample genomes were assembled by mapping to the reference genome from Wuhan, China (hCoV-19/Wuhan/ Hu-1/2019, GenBank accession number: NC_045512.2) using Burrow-Wheeler Aligner (BWA) algorithm embedded in UGENE v. 1.30 [10]. Identification of single nucleotide polymorphisms (SNPs) was performed using the number of high confidence base calls (consensus sequence variations of the assembly) that disagree with the reference bases for the genome position of interest, then all SNPs were exported to a vcf. file and visualized in MS Excel. The following accession IDs for the 17 samples are: EPI_ISL_516800, EPI_ISL_516806, EPI_ISL_516829, EPI_ISL_525492, EPI_ISL_576383, EPI_ISL_632936, EPI_ISL_610161, EPI_ISL_610162, EPI_ISL_576145, EPI_ISL_632937, EPI_ISL_575331, EPI_ISL_576113, EPI_ISL_576114, EPI_ISL_576115, EPI_ISL_576116, EPI_ISL_576128, and EPI_ISL_576130 [11]. The first four IDs have been reported in our previous study [12].

\section{Phylogenetic analysis}

We used the reference genome of hCoV-19/Wuhan/ $\mathrm{Hu}-1 / 2019$ (NC_045512.2) for annotation of our sequences. A dataset of 142 available SARS-CoV-2 genomes (89 sequences from Indonesia and 53 from other countries) was retrieved from GISAID to conduct a phylogenetic analysis (Acknowledgment Table is provided in Additional file 2: Table S2). We only used the full-genome sequences of several strains representing SARS-CoV-2 clades from some countries that had complete genome data and no long stretches of 'NNNN' for the phylogenetic analysis. The MAFFT program server was utilized for multiple nucleotide sequence alignment (https://mafft.cbrc.jp/alignment/server/). A phylogenetic 


\section{(See figure on next page.)}

Fig. 1 Phylogenetic analysis of SARS-CoV-2 genomes from Indonesia and different countries. A phylogenetic tree was constructed from 29.409 nt length of the open reading frame (ORF) of 142 SARS-CoV-2 virus sequences using Neighbor Joining statistical method with 2,000 bootstrap replications. The evolutionary distances were computed using the Kimura 2-parameter method and the rate variation among sites was modelled with a gamma distribution (estimated $a=0.14566$ ). SARS-CoV-2 virus sequences from Indonesia $(N=89)$ followed by the date of collection are indicated in closed circles, while viruses for the study are indicated in red and viruses from the family clusters are color-shaded in yellow. The tree is rooted to Wuhan/Hu-1/2019 with the bootstrap percentage values less than $70 \%$ hidden and it is drawn to scale (0.0001) with branch lengths measured in the number of substitutions per site

tree was constructed from 29.409 nt length of the open reading frame (ORF) of $142 \mathrm{SARS}-\mathrm{CoV}-2$ virus sequences using Neighbor Joining statistical method with 2000 bootstrap replications. The evolutionary distances were computed using the Kimura 2-parameter method and the rate variation among sites was modelled using a gamma distribution with estimated shape parameter $(\alpha)$ for the dataset. The estimation of $\alpha$ gamma distribution was calculated in DAMBE version 7 [13], whereas all the other analyses were performed in MEGA version 10 (MEGA X) [14].

\section{COVID-19 severity classifications}

COVID-19 severity was determined based on the WHO classifications: (1) mild, without evidence of hypoxia or pneumonia; (2) moderate, pneumonia but not severe; (3) severe, pneumonia plus one of the following signs: respiratory rate $>30$ breaths/minute (or based on age for children), severe respiratory distress, or $\mathrm{SpO}_{2}<90 \%$ in room air; and (4) critical, Acute Respiratory Distress Syndrome (ARDS), sepsis, or septic shock, or other complications $[12,15]$.

Our study was approved by the Medical and Health Research Ethics Committee of the Faculty of Medicine, Public Health and Nursing, Universitas Gadjah Mada/ Dr. Sardjito Hospital (KE/FK/0563/EC/2020). All participants or guardians signed a written informed consent for participating in this study.

\section{Results}

\section{Phylogenetic analysis}

Phylogenetic analysis revealed that thirteen virus samples were situated within clade GH (GISAID classification), while two viruses were grouped with other viruses which belonged to clade GR, and one virus each that belonged to clade $\mathrm{O}$ and clade $\mathrm{L}$ (Fig. 1). Three viruses from family cluster case-1 (YO-UGM-10001|EPI_ ISL_576113, YO-UGM-10002|EPI_ISL_576114, and YOUGM-10003|EPI_ISL_576115) formed a single group within clade $\mathrm{GH}$, whereas viruses from family cluster-2 (YO-UGM-1004|EPI_ISL_576116, YO-UGM-1005|EPI_ ISL_576128, and YO-UGM-1006|EPI_ISL_576130,) were separated into two different nodes within clade GH (Fig. 1, top-right).

\section{Molecular analysis}

Ninety-four SNPs were detected throughout the ORP of the SARS-CoV-2 virus samples with $60 \%$ (54/94) of the nucleic acid changes resulting in amino acid substitutions (missense mutations) (Table 1, detailed in Additional file 1: Table S1). The types of nucleic acid base changes were more often detected as transitions (70\%) compared to transversions (30\%). Higher entropy values were observed more from nucleic acids that carried more frequent base changes; however, nucleic acid changes that caused missense mutation could have lower entropy values than those that resulted in synonymous mutation.

The majority of the virus samples (16/17) possessed D614G substitution on spike protein and 56\% of these (9/16) showed other amino acid substitutions on this protein, including L5F, V83L, V213A, W258R, Q677H, and N811I. Second amino acid mutations that were frequently detected were P232L substitution on NSP12 (RdRp) protein (15x), followed by Q57H substitution on NS3 (14x) and P822L substitution on NSP3 protein (13x). Furthermore, various amino acid mutations were also found in the other proteins of virus samples, including on NSP2 (A205V, V247A, T256I, Q321K), NSP3 (P679S, T1022I, A1179V, T1198K, F1354C, P1665L), NSP4 (A231V), NSP5 (K12R, M49I, P184S), NSP6 (L37F), NSP8 (A21T), NSP9 (L42F), NSP12/RdRp (A97V, P227L, T248I, A656S, H892Y, M906V), NSP13 (T127I, T153I, V169F, M576I, P203L), NSP15 (H337Y), NSP16 (Y222C), NS3 (A54V, A99S, T151I, D222Y), NS7a (H73Y), and N (P13L, A119S, Q160R, S193I, R195S, P199S, R203K, G204R, M234I).

\section{COVID-19's severity and spike protein mutations of COVID-19 samples}

Based on the case definition of COVID-19 severity developed for this study, 3 of 17 virus samples (17.6\%) were collected each from asymptomatic cases (people) and critical cases, 5 virus samples (29.4\%) from mild cases, and 6 virus samples (35.3\%) from moderate cases (Table 2). Two of the patients with critical stages eventually died. A range of $\mathrm{Ct}$ values was found amongst different stages of severity, nevertheless all the virus samples with D614G mutations, except one (YO-UGM10004/2020|EPI_ISL_576116), showed lower Ct values 


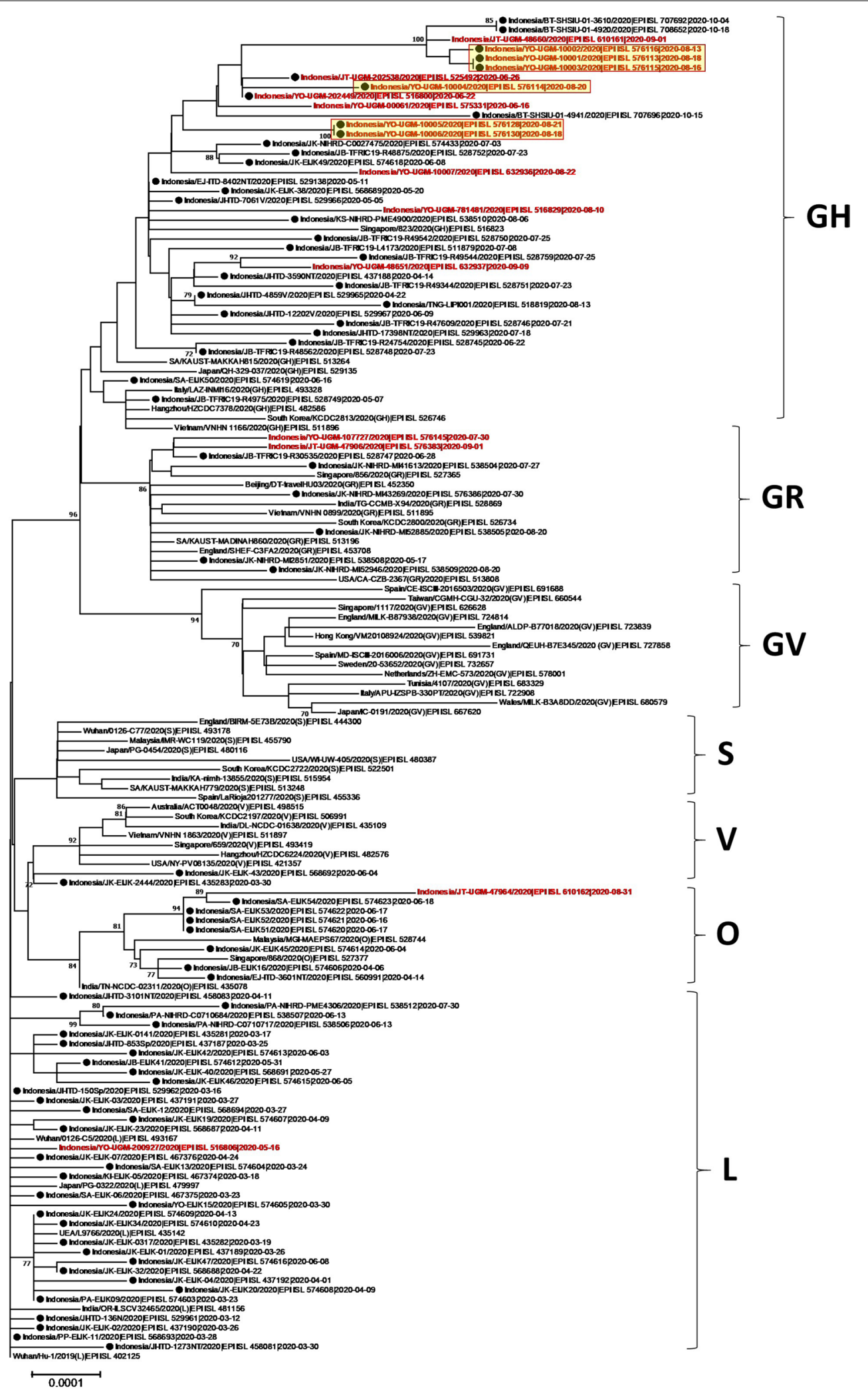


Table 1 Nucleic acid and amino acid mutations observed in seventeen SARS-CoV-2 virus genomes collected from Yogyakarta and Central Java provinces between June and September 2020

\begin{tabular}{|c|c|c|c|c|c|c|c|c|c|}
\hline No. & NA position* & Gene/ Region & Number of SNP & $\begin{array}{l}\text { NA change } \\
\text { (SNP) }\end{array}$ & $\begin{array}{l}\text { Type of base } \\
\text { change }\end{array}$ & Entropy values & Frequency & $\begin{array}{l}\text { Type of } \\
\text { mutation }\end{array}$ & $\begin{array}{l}\text { AA } \\
\text { changes } \\
\text { (position) }\end{array}$ \\
\hline 1 & 1154 & NSP2 & 7 & $\mathrm{C} \rightarrow \mathrm{T}$ & Transition & 0.21456 & 2 & Missense & A205V \\
\hline 2 & 1280 & & & $\mathrm{C} \rightarrow \mathrm{T}$ & Transition & 0.34883 & 2 & Missense & V247A \\
\hline 3 & 1307 & & & $\mathrm{C} \rightarrow \mathrm{T}$ & Transition & 0.45056 & 3 & Missense & T256I \\
\hline 4 & 1501 & & & $C \rightarrow A$ & Transversion & 0.34883 & 2 & Missense & Q321K \\
\hline 5 & 1845 & & & $\mathrm{C} \rightarrow \mathrm{T}$ & Transition & 0.21456 & 1 & Synonymous & $\mathrm{N} 435 \mathrm{~N}$ \\
\hline 6 & 1998 & & & $C \rightarrow T$ & Transition & 0.21456 & 1 & Synonymous & $\mathrm{T} 486 \mathrm{~T}$ \\
\hline 7 & 2247 & & & $A \rightarrow G$ & Transition & 0.21456 & 1 & Synonymous & R569R \\
\hline 8 & 2772 & NSP3 & 13 & $C \rightarrow T$ & Transition & 0.45056 & 15 & Synonymous & F106F \\
\hline 9 & 3264 & & & $\mathrm{~T} \rightarrow \mathrm{C}$ & Transition & 0.21456 & 1 & Synonymous & D270D \\
\hline 10 & 3350 & & & $\mathrm{~T} \rightarrow \mathrm{C}$ & Transition & 0.21456 & 1 & Synonymous & V299A \\
\hline 11 & 3819 & & & $C \rightarrow T$ & Transition & 0.52971 & 4 & Synonymous & G443G \\
\hline 12 & 4489 & & & $\mathrm{C} \rightarrow \mathrm{T}$ & Transition & 0.21456 & 1 & Missense & P679S \\
\hline 13 & 4919 & & & $\mathrm{C} \rightarrow \mathrm{T}$ & Transition & 0.66825 & 13 & Missense & P822L \\
\hline 14 & 5519 & & & $\mathrm{C} \rightarrow \mathrm{T}$ & Transition & 0.21456 & 1 & Missense & T1022I \\
\hline 15 & 5541 & & & $C \rightarrow T$ & Transition & 0.34883 & 2 & Synonymous & C1029C \\
\hline 16 & 5990 & & & $\mathrm{C} \rightarrow \mathrm{T}$ & Transition & 0.21456 & 1 & Missense & A1179V \\
\hline 17 & 6047 & & & $C \rightarrow A$ & Transversion & 0.21456 & 1 & Missense & T1198K \\
\hline 18 & 6515 & & & $\mathrm{~T} \rightarrow \mathrm{G}$ & Transversion & 0.21456 & 1 & Missense & F1354C \\
\hline 19 & 7374 & & & $\mathrm{C} \rightarrow \mathrm{T}$ & Transition & 0.21456 & 1 & Synonymous & F1640F \\
\hline 20 & 7448 & & & $\mathrm{C} \rightarrow \mathrm{T}$ & Transition & 0.21456 & 1 & Missense & P1665L \\
\hline 21 & 8981 & NSP4 & 1 & $\mathrm{C} \rightarrow \mathrm{T}$ & Transition & 0.21456 & 1 & Missense & A231V \\
\hline 22 & 9824 & NSP5 & 4 & $A \rightarrow G$ & Transition & 0.52971 & 4 & Missense & $\mathrm{K} 12 \mathrm{R}$ \\
\hline 23 & 9936 & & & $\mathrm{G} \rightarrow \mathrm{T}$ & Transversion & 0.21456 & 1 & Missense & M49I \\
\hline 24 & 10242 & & & $C \rightarrow T$ & Transition & 0.68696 & 8 & Synonymous & N151N \\
\hline 25 & 10339 & & & $\mathrm{C} \rightarrow \mathrm{T}$ & Transition & 0.21456 & 1 & Missense & P184S \\
\hline 26 & 10818 & NSP6 & 1 & $\mathrm{G} \rightarrow \mathrm{T}$ & Transversion & 0.21456 & 1 & Missense & L37F \\
\hline 27 & 11887 & NSP8 & 2 & $\mathrm{G} \rightarrow \mathrm{A}$ & Transition & 0.21456 & 1 & Missense & A21T \\
\hline 28 & 12174 & & & $C \rightarrow T$ & Transition & 0.21456 & 1 & Synonymous & P116P \\
\hline 29 & 12544 & NSP9 & 1 & $\mathrm{C} \rightarrow \mathrm{T}$ & Transition & 0.21456 & 1 & Missense & L42F \\
\hline 30 & 13465 & NSP12 (RdRp) & 12 & $\mathrm{C} \rightarrow \mathrm{T}$ & Transition & 0.21456 & 1 & Missense & A97V \\
\hline 31 & 13790 & & & $\mathrm{G} \rightarrow \mathrm{T}$ & Transversion & 0.21456 & 1 & Synonymous & L205L \\
\hline 32 & 13855 & & & $\mathrm{C} \rightarrow \mathrm{T}$ & Transition & 0.21456 & 1 & Missense & P227L \\
\hline 33 & 13918 & & & $\mathrm{C} \rightarrow \mathrm{T}$ & Transition & 0.52971 & 3 & Missense & T248I \\
\hline 34 & 14027 & & & $C \rightarrow T$ & Transition & 0.21456 & 1 & Synonymous & D284D \\
\hline 35 & 14143 & & & $\mathrm{C} \rightarrow \mathrm{T}$ & Transition & 0.45056 & 15 & Missense & P323L \\
\hline 36 & 14429 & & & $C \rightarrow T$ & Transition & 0.21456 & 1 & Synonymous & D418D \\
\hline 37 & 15141 & & & $\mathrm{G} \rightarrow \mathrm{T}$ & Transversion & 0.21456 & 1 & Missense & A656S \\
\hline 38 & 15278 & & & $\mathrm{G} \rightarrow \mathrm{T}$ & Transversion & 0.21456 & 1 & Synonymous & T701T \\
\hline 39 & 15500 & & & $A \rightarrow G$ & Transition & 0.21456 & 1 & Synonymous & L775L \\
\hline 40 & 15849 & & & $\mathrm{C} \rightarrow \mathrm{T}$ & Transition & 0.34883 & 2 & Missense & $\mathrm{H} 892 \mathrm{Y}$ \\
\hline 41 & 15891 & & & $A \rightarrow G$ & Transition & 0.21456 & 1 & Missense & M906V \\
\hline 42 & 16130 & NSP13 & 8 & $A \rightarrow T$ & Transversion & 0.45056 & 3 & Synonymous & P53P \\
\hline 43 & 16196 & & & $C \rightarrow T$ & Transition & 0.21456 & 1 & Synonymous & $\mathrm{H} 75 \mathrm{H}$ \\
\hline 44 & 16351 & & & $\mathrm{C} \rightarrow \mathrm{T}$ & Transition & 0.21456 & 1 & Missense & T127I \\
\hline 45 & 16382 & & & $\mathrm{G} \rightarrow \mathrm{T}$ & Transversion & 0.52971 & 4 & Synonymous & T137T \\
\hline 46 & 16429 & & & $\mathrm{C} \rightarrow \mathrm{T}$ & Transition & 0.21456 & 1 & Missense & T153I \\
\hline 47 & 16476 & & & $\mathrm{G} \rightarrow \mathrm{T}$ & Transversion & 0.21456 & 1 & Missense & V169F \\
\hline 48 & 16745 & & & $C \rightarrow T$ & Transition & 0.21456 & 1 & Synonymous & $|258|$ \\
\hline 49 & 17699 & & & $\mathrm{G} \rightarrow \mathrm{T}$ & Transversion & 0.21456 & 1 & Missense & M576I \\
\hline
\end{tabular}


Table 1 (continued)

\begin{tabular}{|c|c|c|c|c|c|c|c|c|c|}
\hline No. & NA position* & Gene/ Region & Number of SNP & $\begin{array}{l}\text { NA change } \\
\text { (SNP) }\end{array}$ & $\begin{array}{l}\text { Type of base } \\
\text { change }\end{array}$ & Entropy values & Frequency & $\begin{array}{l}\text { Type of } \\
\text { mutation }\end{array}$ & $\begin{array}{l}\text { AA } \\
\text { changes } \\
\text { (position) }^{\#}\end{array}$ \\
\hline 50 & 18382 & NSP14 & 4 & $\mathrm{C} \rightarrow \mathrm{T}$ & Transition & 0.21456 & 1 & Missense & P203L \\
\hline 51 & 18479 & & & $\mathrm{C} \rightarrow \mathrm{T}$ & Transition & 0.68696 & 8 & Synonymous & Y235Y \\
\hline 52 & 18612 & & & $C \rightarrow T$ & Transition & 0.59084 & 14 & Synonymous & L280L \\
\hline 53 & 18737 & & & $A \rightarrow G$ & Transition & 0.21456 & 1 & Synonymous & L321L \\
\hline 54 & 19859 & NSP15 & 2 & $\mathrm{~T} \rightarrow \mathrm{C}$ & Transition & 0.21456 & 1 & Synonymous & $|168|$ \\
\hline 55 & 20364 & & & $\mathrm{C} \rightarrow \mathrm{T}$ & Transition & 0.21456 & 1 & Missense & $H 337 Y$ \\
\hline 56 & 20843 & NSP16 & 2 & $C \rightarrow T$ & Transition & 0.21456 & 1 & Synonymous & F150F \\
\hline 57 & 21058 & & & $A \rightarrow G$ & Transition & 0.21456 & 2 & Missense & $\mathrm{Y} 222 \mathrm{C}$ \\
\hline 58 & 21310 & Spike (S) & 12 & $\mathrm{C} \rightarrow \mathrm{T}$ & Transition & 0.34883 & 2 & Missense & L5F \\
\hline 59 & 21387 & & & $\mathrm{~T} \rightarrow \mathrm{C}$ & Transition & 0.21456 & 1 & Synonymous & N30N \\
\hline 60 & 21477 & & & $C \rightarrow T$ & Transition & 0.52971 & 4 & Synonymous & S60S \\
\hline 61 & 21483 & & & $\mathrm{~T} \rightarrow \mathrm{C}$ & Transition & 0.21456 & 1 & Synonymous & V62V \\
\hline 62 & 21544 & & & $\mathrm{G} \rightarrow \mathrm{C}$ & Transversion & 0.21456 & 1 & Missense & V83L \\
\hline 63 & 21935 & & & $\mathrm{~T} \rightarrow \mathrm{C}$ & Transition & 0.52971 & 4 & Missense & V213A \\
\hline 64 & 22069 & & & $\mathrm{~T} \rightarrow \mathrm{C}$ & Transition & 0.21456 & 1 & Missense & W258R \\
\hline 65 & 23138 & & & $A \rightarrow G$ & Transition & 0.34883 & 16 & Missense & D614G \\
\hline 66 & 23328 & & & $\mathrm{G} \rightarrow \mathrm{T}$ & Transversion & 0.21456 & 1 & Missense & Q677H \\
\hline 67 & 23664 & & & $C \rightarrow T$ & Transition & 0.21456 & 1 & Synonymous & Y789Y \\
\hline 68 & 23729 & & & $A \rightarrow T$ & Transversion & 0.21456 & 1 & Missense & N811I \\
\hline 69 & 23928 & & & $\mathrm{G} \rightarrow \mathrm{T}$ & Transversion & 0.21456 & 1 & Synonymous & L877L \\
\hline 70 & 25288 & NS3 & 6 & $\mathrm{C} \rightarrow \mathrm{T}$ & Transition & 0.21456 & 1 & Missense & A54V \\
\hline 71 & 25298 & & & $G \rightarrow T$ & Transversion & 0.59084 & 14 & Missense & Q57H \\
\hline 72 & 25349 & & & $C \rightarrow T$ & Transition & 0.34883 & 2 & Synonymous & S74S \\
\hline 73 & 25422 & & & $\mathrm{G} \rightarrow \mathrm{T}$ & Transversion & 0.21456 & 1 & Missense & A99S \\
\hline 74 & 25579 & & & $\mathrm{C} \rightarrow \mathrm{T}$ & Transition & 0.21456 & 1 & Missense & T151I \\
\hline 75 & 25791 & & & $\mathrm{G} \rightarrow \mathrm{T}$ & Transversion & 0.21456 & 1 & Missense & D222Y \\
\hline 76 & 26470 & M & 3 & $C \rightarrow T$ & Transition & 0.59084 & 14 & Synonymous & Y71Y \\
\hline 77 & 26536 & & & $C \rightarrow A$ & Transversion & 0.34883 & 2 & Synonymous & L93L \\
\hline 78 & 26602 & & & $A \rightarrow G$ & Transition & 0.66825 & 7 & Synonymous & E115E \\
\hline 79 & 27345 & NS7a & 1 & $\mathrm{C} \rightarrow \mathrm{T}$ & Transition & 0.21456 & 1 & Missense & $\mathrm{H} 73 \mathrm{Y}$ \\
\hline 80 & 27808 & NS8 & 1 & $\mathrm{G} \rightarrow \mathrm{A}$ & Transition & 0.21456 & 1 & Synonymous & L60L \\
\hline 81 & 28046 & N & 14 & $\mathrm{C} \rightarrow \mathrm{T}$ & Transition & 0.21456 & 1 & Missense & P13L \\
\hline 82 & 28363 & & & $\mathrm{G} \rightarrow \mathrm{T}$ & Transversion & 0.52971 & 4 & Missense & A119S \\
\hline 83 & 28470 & & & $\mathrm{~T} \rightarrow \mathrm{C}$ & Transition & 0.21456 & 1 & Synonymous & N154N \\
\hline 84 & 28479 & & & $C \rightarrow T$ & Transition & 0.21456 & 1 & Synonymous & |157| \\
\hline 85 & 28487 & & & $A \rightarrow G$ & Transition & 0.21456 & 1 & Missense & Q160R \\
\hline 86 & 28586 & & & $\mathrm{G} \rightarrow \mathrm{T}$ & Transversion & 0.52971 & 4 & Missense & S193I \\
\hline 87 & 28593 & & & $A \rightarrow T$ & Transversion & 0.21456 & 1 & Missense & R195S \\
\hline 88 & 28603 & & & $\mathrm{C} \rightarrow \mathrm{T}$ & Transition & 0.21456 & 1 & Missense & P199S \\
\hline 89 & 28616 & & & $\mathrm{G} \rightarrow \mathrm{A}$ & Transition & 0.34883 & 2 & Missense & R203K \\
\hline 90 & 28617 & & & $G \rightarrow A$ & Transition & 0.34883 & 2 & & \\
\hline 91 & 28618 & & & $\mathrm{G} \rightarrow \mathrm{C}$ & Transversion & 0.34883 & 2 & Missense & G204R \\
\hline 92 & 28710 & & & $\mathrm{G} \rightarrow \mathrm{T}$ & Transversion & 0.21456 & 1 & Missense & M234I \\
\hline 93 & 28944 & & & $A \rightarrow G$ & Transition & 0.21456 & 1 & Synonymous & S312S \\
\hline 94 & 29094 & & & $A \rightarrow T$ & Transversion & 0.34883 & 2 & Synonymous & T362T \\
\hline
\end{tabular}

Bold indicates non-synonymous substitutions

*Nucleic acid numbering starting from ORF1ab start codon (ATG)

\# Amino acid numbering starting from start codon of each gene 
Table 2 Severity and genetic data associated with SARS-CoV-2 viruses collected from seventeen COVID-19 patients in Yogyakarta and Central Java provinces, Indonesia from June-September 2020

\begin{tabular}{|c|c|c|c|c|c|c|c|c|c|}
\hline Patient no. & Sex & Age (yo) & $\begin{array}{l}\text { COVID-19 } \\
\text { severity }\end{array}$ & $C_{T}$ value & $\begin{array}{l}\text { Virus name (GISAID } \\
\text { Accession ID) }\end{array}$ & $\begin{array}{l}\text { Average } \\
\text { coverage }\end{array}$ & Collection date & $\begin{array}{l}\text { Lineage } \\
\text { (GISAID } \\
\text { clade) }\end{array}$ & $\begin{array}{l}\text { Spike } \\
\text { mutations }\end{array}$ \\
\hline 1 & Male & 30 & Mild & 27.9 & $\begin{array}{l}\text { hCoV19/Indonesia/YO- } \\
\text { UGM-200927/2020 } \\
\text { (EPI_ISL_516806) }\end{array}$ & $102 x$ & $16 / 05 / 2020$ & L & - \\
\hline 2 & Female & 49 & Asymptomatic & 22.31 & $\begin{array}{l}\text { hCoV-19/Indonesia/ } \\
\text { YO-UGM-00061/2020 } \\
\text { (EPI_ISL_575331) }\end{array}$ & $4655 x$ & $16 / 06 / 2020$ & $\mathrm{GH}$ & D614G \\
\hline 3 & Male & 77 & Moderate & 19.7 & $\begin{array}{l}\text { hCoV19/Indonesia/YO- } \\
\text { UGM-202449/2020 } \\
\text { (EPI_ISL_516800) }\end{array}$ & $22088 x$ & $22 / 06 / 2020$ & $\mathrm{GH}$ & D614G \\
\hline 4 & Female & 55 & Moderate & 24.7 & $\begin{array}{l}\text { hCov19/Indonesia/ } \\
\text { JT-UGM-202538/2020 } \\
\text { (EPI_ISL_525492) }\end{array}$ & $347 x$ & $26 / 06 / 2020$ & $\mathrm{GH}$ & D614G \\
\hline 5 & Male & 28 & Asymptomatic & 21.05 & $\begin{array}{l}\text { hCoV-19/Indonesia/YO- } \\
\text { UGM-107727/2020 } \\
\text { (EPI_ISL_576145) }\end{array}$ & $104 x$ & $30 / 07 / 2020$ & $G R$ & D614G \\
\hline 6 & Female & 83 & Moderate & 16.9 & $\begin{array}{l}\text { hCoV19/Indonesia/YO- } \\
\text { UGM-781481/2020 } \\
\text { (EPI_ISL_516829) }\end{array}$ & $3748 x$ & $10 / 08 / 2020$ & $\mathrm{GH}$ & D614G \\
\hline 7 & Male & 28 & Critical & 20 & $\begin{array}{l}\text { hCoV-19/Indonesia/ } \\
\text { YO-UGM-10002/2020 } \\
\text { (EPI_ISL_576114)* }\end{array}$ & $13 x$ & $13 / 08 / 2020$ & $\mathrm{GH}$ & V213A, D614G \\
\hline 8 & Male & 58 & Critical (Died) & 23 & $\begin{array}{l}\text { hCoV-19/Indonesia/ } \\
\text { YO-UGM-10003/2020 } \\
\text { (EPI_ISL_576115)* }\end{array}$ & $505 x$ & $16 / 08 / 2020$ & $\mathrm{GH}$ & V213A, D614G \\
\hline 9 & Male & 88 & Critical (Died) & 18.1 & $\begin{array}{l}\text { hCoV-19/Indonesia/ } \\
\text { YO-UGM-10001/2020 } \\
\text { (EPI_ISL_576113)* }\end{array}$ & $42888 x$ & $18 / 08 / 2020$ & $\mathrm{GH}$ & V213A, D614G \\
\hline 10 & Male & 8 & Moderate & 19.5 & $\begin{array}{l}\text { hCoV-19/Indonesia/ } \\
\text { YO-UGM-10006/2020 } \\
\text { (EPI_ISL_576130*** }\end{array}$ & $26 x$ & 18/08/2020 & $\mathrm{GH}$ & L5F, D614G \\
\hline 11 & Male & 35 & Mild & 32 & $\begin{array}{l}\text { hCoV-19/Indonesia/ } \\
\text { YO-UGM-10004/2020 } \\
(\text { EPI_ISL_576116)** }\end{array}$ & $45 x$ & $20 / 08 / 2020$ & $\mathrm{GH}$ & D614G \\
\hline 12 & Female & 33 & Mild & 22 & $\begin{array}{l}\text { hCoV-19/Indonesia/ } \\
\text { YO-UGM-10005/2020 } \\
\text { (EPI_ISL_576128)** }\end{array}$ & $106 x$ & $21 / 08 / 2020$ & $\mathrm{GH}$ & L5F, D614G \\
\hline 13 & Female & 52 & Asymptomatic & 18 & $\begin{array}{l}\text { hCoV-19/Indonesia/ } \\
\text { YO-UGM-10007/2020 } \\
\text { (EPI_ISL_632936) }\end{array}$ & $2207 x$ & $22 / 08 / 2020$ & $\mathrm{GH}$ & D614G, N811I \\
\hline 14 & Male & 48 & Moderate & 19.64 & $\begin{array}{l}\text { hCoV-19/Indonesia/ } \\
\text { JT-UGM-47964/2020 } \\
\text { (EPI_ISL_610162) }\end{array}$ & $6291 x$ & $31 / 08 / 2020$ & $\mathrm{O}$ & W258R, D614G \\
\hline 15 & Male & 36 & Mild & 17.53 & $\begin{array}{l}\text { hCoV-19/Indonesia/ } \\
\text { JT-UGM-47906/2020 } \\
\text { (EPI_ISL_576383) }\end{array}$ & $653 x$ & $01 / 09 / 2020$ & $\mathrm{GR}$ & D614G \\
\hline 16 & Female & 64 & Moderate & 19.44 & $\begin{array}{l}\text { hCoV-19/Indonesia/ } \\
\text { JT-UGM-48660/2020 } \\
\text { (EPI_ISL_610161) }\end{array}$ & $22 x$ & $01 / 09 / 2020$ & $\mathrm{GH}$ & V213A, D614G \\
\hline 17 & Male & 41 & Mild & 21.24 & $\begin{array}{l}\text { hCoV-19/Indonesia/ } \\
\text { YO-UGM-48651/2020 } \\
\text { (EPI_ISL_632937) }\end{array}$ & $2867 x$ & 09/09/2020 & $\mathrm{GH}$ & $\begin{array}{l}\text { V83L, D614G, } \\
\text { Q677H }\end{array}$ \\
\hline
\end{tabular}

Virus samples collected from family cluster- ${ }^{*}$ ) and from family cluster-2**)

CT, cycle threshold; Ref. sequence: hCoV-19/Wuhan/Hu-1/2019 (NC_045512.2) 


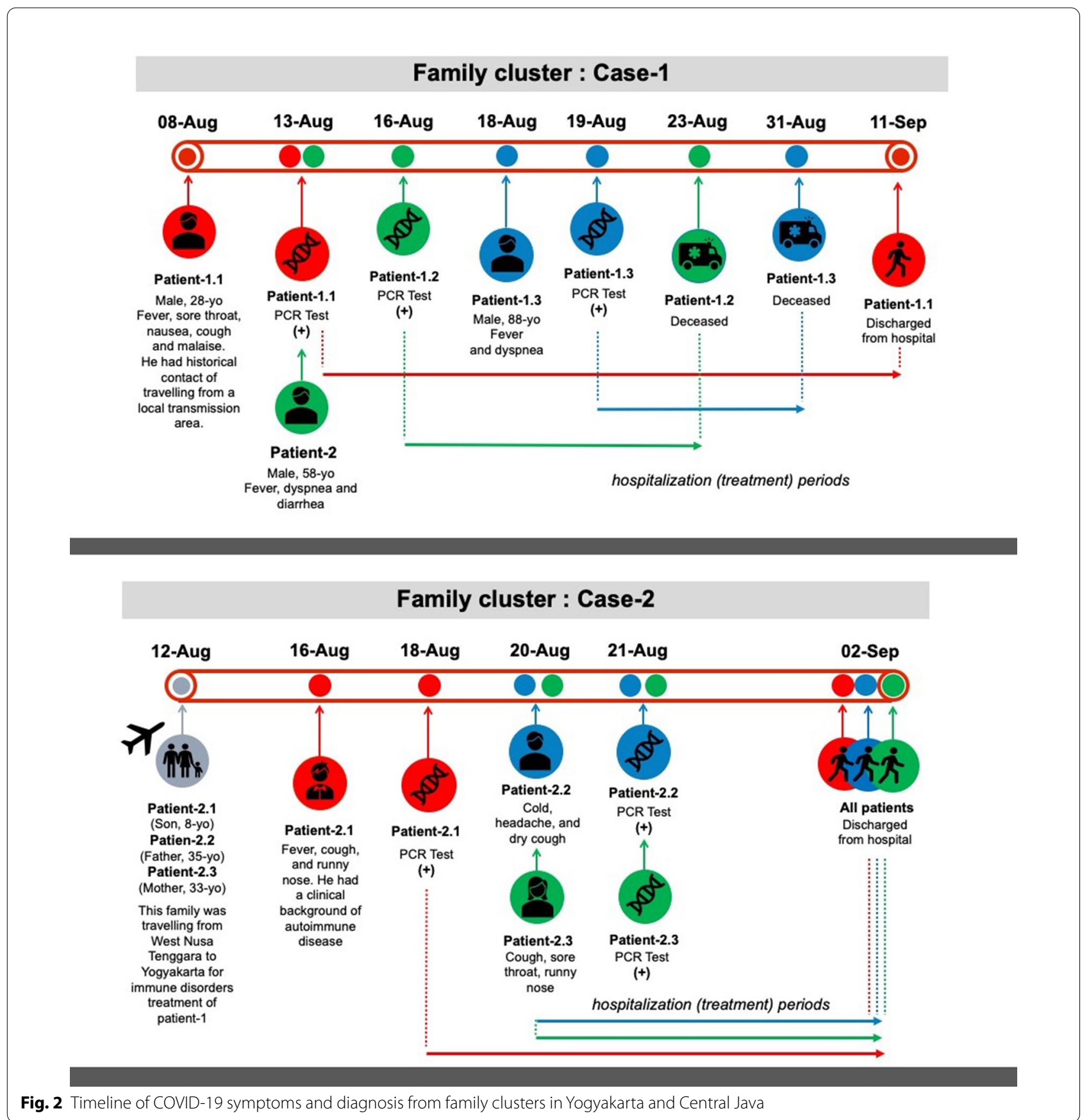

(clade GH, GR, and $\mathrm{O}, \mathrm{Ct}$ range 16.9-24.7) than those with no mutation in this position (clade L, Ct 27.9). Dual mutations of V213A and D614G on spike protein were detected in four patients, and two of these eventually died after a period of hospitalization.

\section{Disease outcomes of COVID-19's family clusters}

The epidemiological and clinical data of COVID-19's family clusters, including clinical symptoms, date of first symptoms appeared, diagnostic results, abnormal findings, comorbidity background are provided by timeline and tabulation in Fig. 2 and Table 3, respectively.

In family cluster-1, all three patients showed critical COVID-19 and eventually, two died (YO-UGM-10001|EPI_ISL_576113 and YOUGM-10003|EPI_ISL_576115) and one survived (YOUGM-10002|EPI_ISL_576114). The disease began from patient-1.1, a 28-year-old male, who had a history of 
Table 3 Characteristics of patients with COVID-19 from family cluster cases in Yogyakarta and Central Java

\begin{tabular}{|c|c|c|c|c|c|c|c|c|}
\hline Patient no. & Sex & Age (yo) & $\begin{array}{l}\text { COVID-19 } \\
\text { severity }\end{array}$ & Symptoms & $\begin{array}{l}\text { Date of first } \\
\text { symptoms } \\
\text { appeared }\end{array}$ & $\begin{array}{l}\text { Abnormal } \\
\text { findings }\end{array}$ & Comorbidity & $\begin{array}{l}\text { Virus name } \\
\text { (GISAID Accession } \\
\text { ID) }\end{array}$ \\
\hline 1.1 & Male & 28 & Critical & $\begin{array}{l}\text { Fever, sore throat, } \\
\text { nausea, cough, } \\
\text { malaise }\end{array}$ & 08/08/2020 & $\begin{array}{l}\text { CXR: typical } \\
\text { COVID-19 } \\
\text { bilateral pneu- } \\
\text { monia } \\
\text { NLR } 12.2 \\
\text { ARDS PaO2/FIO2 } \\
260\end{array}$ & Obesity & $\begin{array}{l}\text { hCoV-19/ } \\
\text { Indonesia/YO- } \\
\text { UGM-10002/2020 } \\
\text { (EPI_ISL_576114) }\end{array}$ \\
\hline 1.2 & Male & 58 & Critical (Died) & $\begin{array}{l}\text { Fever, dyspnea, } \\
\text { diarrhea }\end{array}$ & $13 / 08 / 2020$ & $\begin{array}{l}\text { Crackles in both } \\
\text { lungs } \\
\text { NLR } 8.2 \\
\text { 5th days ARDS } \\
\text { PaO2/FIO2 } 68 \\
\text { (intubated) }\end{array}$ & $\begin{array}{l}\text { DM, Hyperten- } \\
\text { sion and } \\
\text { obesity }\end{array}$ & $\begin{array}{l}\text { hCoV-19/ } \\
\text { Indonesia/YO- } \\
\text { UGM-10003/2020 } \\
\text { (EPI_ISL_576115) }\end{array}$ \\
\hline 1.3 & Male & 88 & Critical (Died) & Fever, dyspnea & 19/08/2020 & $\begin{array}{l}\text { Crackles in both } \\
\text { lungs } \\
\text { CXR: bilateral } \\
\text { pneumonia } \\
\text { and cardio- } \\
\text { megaly } \\
\text { Electrolyte imbal- } \\
\text { ance, PaO2/ } \\
\text { FIO2 123.3 } \\
\text { (worsening } \\
\text { ARDS) } \\
\text { Intubated on 7th } \\
\text { day }\end{array}$ & $\begin{array}{l}\text { Type } 2 \text { DM, } \\
\text { Geriatric syn- } \\
\text { drome, History } \\
\text { of infarction } \\
\text { stroke }\end{array}$ & $\begin{array}{l}\text { hCoV-19/ } \\
\text { Indonesia/YO- } \\
\text { UGM-10001/2020 } \\
\text { (EPI_ISL_576113) }\end{array}$ \\
\hline 2.1 & Male & 8 & Moderate & $\begin{array}{c}\text { Fever, cough, } \\
\text { runny nose }\end{array}$ & $16 / 08 / 2020$ & $\begin{array}{l}\text { CXR: bilateral } \\
\text { paracardial } \\
\text { infiltrate }\end{array}$ & $\begin{array}{l}\text { Autoimmune } \\
\text { disorders } \\
\text { (Henoch- } \\
\text { Schönlein } \\
\text { purpura) }\end{array}$ & $\begin{array}{l}\text { hCoV-19/ } \\
\text { Indonesia/YO- } \\
\text { UGM-10006/2020 } \\
\text { (EPI_ISL_576130) }\end{array}$ \\
\hline 2.2 & Male & 35 & Mild & $\begin{array}{l}\text { Cold, headache, } \\
\text { dry cough }\end{array}$ & $20 / 08 / 2020$ & $\begin{array}{l}\text { Eosinophilia } \\
(6.4 \%) \\
\mathrm{pH}(7.45), \mathrm{PaO} 2 \\
(83.2), \mathrm{PaCO} 2 \\
\text { (39.2), } \mathrm{SaO} 2 \\
\text { (96.8), } \mathrm{PaO} 2 / \\
\mathrm{FiO2}(416)\end{array}$ & Hypertension & $\begin{array}{l}\text { hCoV-19/ } \\
\text { Indonesia/YO- } \\
\text { UGM-10004/2020 } \\
\text { (EPI_ISL_576116) }\end{array}$ \\
\hline 2.3 & Female & 33 & Mild & $\begin{array}{l}\text { Cough, sore } \\
\text { throat, runny } \\
\text { nose }\end{array}$ & $20 / 08 / 2020$ & No abnormality & None & $\begin{array}{l}\text { hCoV-19/ } \\
\text { Indonesia/YO- } \\
\text { UGM-10005/2020 } \\
\text { (EPI_ISL_576128) }\end{array}$ \\
\hline
\end{tabular}

CXR chest X-ray, ARDS acute respiratory distress syndrome, NLR neutrophil to lymphocyte ratio, DM diabetes mellitus

traveling from the local COVID-19 transmission area. He complained of fever, sore throat, cough and malaise on August 8th, 2020, and was tested for PCR three days afterward with the result of COVID-19 positive. His father (patient-1.2, 58-yo), who was living in the same house, showed fever, dyspnea and diarrhea on August 13th, then was followed by his grandfather (patient-1.3, 88-yo) who showed fever and dyspnea on August 18th. The PCR tests for both patients showed positive for COVID-19. All patients developed severe disease outcomes including bilateral pneumonia, cardiomegaly and ARDS. Several comorbidities were recorded from patient-1.1 (obesity), patient-1.2 (diabetes mellitus, and obesity), and patient-1.3 (type 2 diabetes mellitus, geriatric syndrome, history of infarction stroke). Patient-1.1 was uneventfully discharged from the hospital on day 29 of hospitalization, but sadly, patient-1.2 and patient-1.3 passed away in the hospital after 7 and 12 days of hospitalization, respectively.

Family cluster-2 involved three patients which were comprised of a son, 8-yo (patient-2.1), father, 35-yo (patient-2.2) and mother, 33-yo, with the following virus samples: YO-UGM-10006 (EPI_ISL_576130), YO-UGM-10004/2020 (EPI_ISL_576114), and 
YO-UGM-10005/2020 (EPI_ISL_576128), respectively. Prior to the index case, this family travelled from West Nusa Tenggara to Yogyakarta on August 2nd, 2020, in order to obtain medical treatment for patient-2.1 who had an autoimmune disorder in a hospital in Yogyakarta. Patient-2.1 had firstly exhibited symptoms of fever, cough, and runny nose on August 11th, 2020 and he was diagnosed COVID-19 positive on August 18th. His parents showed clinical signs of cold, headache and dry cough (patient-2.2) and cough, sore throat, and runny nose (patient-2.3) at the same day on August 20th and the PCR results of both patients were positive on August 21st. Patient-2.1 developed moderate severity with bilateral paracardial infiltrate, whereas patient-2.2 and patient-2.3 developed mild disease without any abnormalities in their chest X-rays and other laboratory findings, except eosinophilia (6.4\%), increased levels in $\mathrm{pH}$ of the blood (7.45), $\mathrm{PaO}_{2}$ (83.2), $\mathrm{PaCO}_{2}$ (39.2) with $\mathrm{SaO}_{2}$ 96.8\% and $\mathrm{PaO}_{2} / \mathrm{FiO}_{2}$ value was 416 from the arterial blood glass analysis of patient-2.2. All three patients uneventfully recovered and were discharged from the hospital on September 2nd, 2020.

\section{Molecular characterizations of virus samples collected from family clusters}

Phylogenetic analysis revealed that the three viruses from family cluster-1 were grouped together from a single node. A matrix of nucleic acid difference showed that YO-UGM-10001|EPI_ISL_576113 and YO-UGM10003|EPI_ISL_576115 were identical on their ORF (nucleic acid and protein levels) and both virus strains had differences of 2 nucleic acids and 1 amino acid in the NSP2 protein which correspond with V247A substitution in YO-UGM-10001|EPI_ISL_576113 and YOUGM-10003|EPI_ISL_576115 and T256I substitution in YO-UGM-10002|EPI_ISL_576114, respectively (Table 4). Other unique mutations in the other viral proteins were detected in these three virus strains which were not shown in the other study viruses, including V213A (Spike), K12R (NSP5), T248I (NSP12/RdRp), A119S and S193I (N). The virus samples from family cluster- 2 were separated in different nodes in the phylogenetic tree (Fig. 3). The tree and the matrix sequence showed that YO-UGM10005/2020|EPI_ISL_576128 and YO-UGM-10006|EPI_ ISL_576130 were genetically identical. Both virus strains had 15 nucleic acid differences compared to YO-UGM10004|EPI_ISL_576114 which resulted in amino acid variations detected in several viral proteins (Table 4).

\section{Discussion}

Our study provides evidence of SARS-CoV-2 transmission within families, in which the same mutation of the spike protein in each family cluster was identified. It is important to understand the transmission routes of SARS-CoV-2 to prevent and control its spreading [4]. Families have been reported as the most dominant infection cluster of COVID-19 [16]. Family clusters have a higher risk of cross-infection because of frequent and close contact among each family member [4]. Our study also documented that although all family members showed the same multiple $S$ protein mutations, however, they revealed different outcomes. While multiple $\mathrm{S}$ protein mutations, i.e. B.1.1.7 variant, have been associated with the severity of COVID-19 [17, 18], this is not the case for our patients. Our samples did not consist of B.1.1.7 variant. In addition, several prognostic factors have been associated with increased risk of severity and mortality of COVID-19, including increasing age, obesity, and comorbidities such as hypertension, diabetes and cerebrovascular disease [15]. Our patients who eventually died (YO-UGM-10001|EPI_ISL_576113 and YO-UGM10003|EPI_ISL_576115) have more prognostic factors than the patient who survived (YO-UGM-10002|EPI ISL_576114) (Table 3). Besides the SARS-CoV-2 variants and prognostic factors, a recent GWAS identified rs11385942 at locus 3p21.31 and rs657152 at locus 9q34.2 as a genetic risk factor for severe COVID-19 [19]. Further study is necessary to confirm whether these polymorphisms might be as susceptible factors in our patients.

Double mutations of V213A and D614G on spike protein were detected in four patients, but three of them (75\%) developed severe diseases causing critical conditions and two (50\%) with fatal outcome. Another interesting finding was documented from family cluster-1, in which all the virus samples in this family cluster belong to the same clade $\mathrm{GH}$, but two patients died and one survived (Table 2). All samples from family cluster-1 revealed another spike protein mutation, V213A, besides D614G. However, virus samples isolated from fatal disease outcomes carried V247A mutation in the NSP2 protein, while those from the recovered patient did not. In conjunction with D614G mutation, substitution of valine $(\mathrm{V})$ to alanine $(\mathrm{A})$ in position 247 and 213 of NSP2 and spike protein, respectively, were detected in the patients with fatal disease outcomes. While both $\mathrm{V}$ and $\mathrm{A}$, as well as $\mathrm{G}$ are in the non-polar hydrophobic amino acid group and no evidence shows that the double mutations of V213A and D614G affect the severity and lethality of COVID-19 patients, further investigations are necessary to determine whether these dual mutations (V213A and D614G in spike protein) or even triple mutations (V213A and D614G in spike protein and V47A in NS2) associated with increased risk of mortality in COVID-19 patients. Moreover, due to limited number of sample size in this study, it is very difficult to associate between the number of mutations on 
Table 4 Amino acid mutations detected in SARS-CoV-2 viruses collected from two family cluster cases in Yogyakarta and Central Java provinces

\begin{tabular}{|c|c|c|c|c|c|c|c|c|c|c|}
\hline \multirow{2}{*}{$\begin{array}{l}\text { Virus (Accession No.) } \\
\text { Amino acid position in each gene }\end{array}$} & \multicolumn{3}{|l|}{ NSP2 } & \multicolumn{2}{|c|}{ NSP3 } & \multirow{2}{*}{$\begin{array}{l}\text { NSP4 } \\
231\end{array}$} & \multirow{2}{*}{$\begin{array}{l}\text { NSP5 } \\
12\end{array}$} & \multicolumn{3}{|c|}{ NSP12 (RdRp) } \\
\hline & 247 & 256 & 321 & 299 & 822 & & & 248 & 323 & 892 \\
\hline Wuhan/19 (NC_045512.2) & V & $T$ & Q & V & $P$ & $A$ & K & $T$ & P & $H$ \\
\hline YO-UGM-10001 (EPI_ISL_576113)* & A & $\mathrm{T}$ & Q & V & $\mathbf{L}$ & A & $\mathbf{R}$ & I & $\mathbf{L}$ & $\mathrm{H}$ \\
\hline YO-UGM-10002 (EPI_ISL_576114)* & V & I & Q & V & $\mathbf{L}$ & A & $\mathbf{R}$ & I & $\mathbf{L}$ & $\mathrm{H}$ \\
\hline YO-UGM-10003 (EPI_ISL_576115)* & A & T & Q & V & L & A & $\mathbf{R}$ & I & $\mathbf{L}$ & $\mathrm{H}$ \\
\hline YO-UGM-10004 (EPI_ISL_576116)** & V & $\mathrm{T}$ & Q & A & $\mathbf{L}$ & v & K & $\mathrm{T}$ & $\mathbf{L}$ & $\mathrm{H}$ \\
\hline YO-UGM-10005 (EPI_ISL_576128)** & V & I & K & V & $\mathbf{L}$ & A & K & $\mathrm{T}$ & $\mathbf{L}$ & $\mathbf{Y}$ \\
\hline YO-UGM-10006 (EPI_ISL_576130)** & V & 1 & K & V & $\mathbf{L}$ & A & K & $\mathrm{T}$ & $\mathbf{L}$ & $\mathrm{Y}$ \\
\hline YO-UGM-10007 (EPI_ISL_632936) & V & $\mathrm{T}$ & Q & V & $\mathbf{L}$ & A & K & $\mathrm{T}$ & $\mathbf{L}$ & $\mathrm{H}$ \\
\hline YO-UGM-781481 (EPI_ISL_516829) & V & $\mathrm{T}$ & Q & V & P & A & K & $\mathrm{T}$ & $\mathbf{L}$ & $\mathrm{H}$ \\
\hline YO-UGM-202449 (EPI_ISL_516800) & V & $\mathrm{T}$ & Q & V & $\mathbf{L}$ & A & K & $\mathrm{T}$ & $\mathbf{L}$ & $\mathrm{H}$ \\
\hline JT-UGM-202538 (EPI_ISL_525492) & V & $\mathrm{T}$ & Q & V & L & A & K & $\mathrm{T}$ & $\mathbf{L}$ & $\mathrm{H}$ \\
\hline YO-UGM-200927 (EPI_ISL_516806) & V & $\mathrm{T}$ & Q & V & $P$ & A & K & $\mathrm{T}$ & $P$ & $\mathrm{H}$ \\
\hline JT-UGM-47906 (EPI_ISL_576383) & V & $\mathrm{T}$ & Q & V & $P$ & A & K & $\mathrm{T}$ & $\mathbf{L}$ & $\mathrm{H}$ \\
\hline JT-UGM-47964 (EPI_ISL_610162) & V & $\mathrm{T}$ & Q & V & $P$ & A & K & $\mathrm{T}$ & $P$ & $\mathrm{H}$ \\
\hline JT-UGM-48660 (EPI_ISL_610161) & V & $\mathrm{T}$ & Q & V & $\mathbf{L}$ & A & $\mathbf{R}$ & $\mathrm{T}$ & $\mathbf{L}$ & $\mathrm{H}$ \\
\hline YO-UGM-00061 (EPI_ISL_575331) & V & $\mathrm{T}$ & Q & V & $\mathbf{L}$ & A & K & $\mathrm{T}$ & $\mathbf{L}$ & $\mathrm{H}$ \\
\hline YO-UGM-48651 (EPI_ISL_632937) & V & $\mathrm{T}$ & Q & V & $P$ & A & K & $\mathrm{T}$ & $\mathbf{L}$ & $\mathrm{H}$ \\
\hline YO-UGM-107727 (EPI_ISL_576145) & V & $\mathrm{T}$ & Q & V & $P$ & A & K & $\mathrm{T}$ & $\mathbf{L}$ & $\mathrm{H}$ \\
\hline Virus (Accession No.) & NSP13 & NSP14 & Spike & & & NS3 & $N$ & & & \\
\hline Amino acid position in each gene & & & 5 & 213 & 614 & 57 & 119 & 193 & & \\
\hline Wuhan/19 (NC_045512.2) & $T$ & $P$ & L & V & $D$ & Q & $A$ & $S$ & & \\
\hline YO-UGM-10001 (EPI_ISL_576113)* & $\mathrm{T}$ & $P$ & L & A & G & $\mathbf{H}$ & $\mathrm{S}$ & I & & \\
\hline YO-UGM-10002 (EPI_ISL_576114)* & $\mathrm{T}$ & $P$ & $\mathrm{~L}$ & A & G & $\mathbf{H}$ & $\mathbf{S}$ & 1 & & \\
\hline YO-UGM-10003 (EPI_ISL_576115)* & $\mathrm{T}$ & $P$ & $\mathrm{~L}$ & A & G & $\mathbf{H}$ & $\mathbf{S}$ & I & & \\
\hline YO-UGM-10004 (EPI_ISL_576116)** & 1 & $\mathbf{L}$ & $\mathrm{L}$ & V & G & $\mathbf{H}$ & A & $\mathrm{S}$ & & \\
\hline YO-UGM-10005 (EPI_ISL_576128)** & $\mathrm{T}$ & $P$ & $\mathbf{F}$ & V & G & $\mathbf{H}$ & A & S & & \\
\hline YO-UGM-10006 (EPI_ISL_576130)** & $\mathrm{T}$ & $P$ & $\mathbf{F}$ & V & G & $\mathbf{H}$ & A & S & & \\
\hline YO-UGM-10007 (EPI_ISL_632936) & $\mathrm{T}$ & $P$ & $\mathrm{~L}$ & V & G & $\mathrm{H}$ & A & $\mathrm{S}$ & & \\
\hline YO-UGM-781481 (EPI_ISL_516829) & $\mathrm{T}$ & $P$ & $\mathrm{~L}$ & V & G & $\mathrm{H}$ & A & $\mathrm{S}$ & & \\
\hline YO-UGM-202449 (EPI_ISL_516800) & $\mathrm{T}$ & $P$ & $\mathrm{~L}$ & V & G & $\mathbf{H}$ & A & S & & \\
\hline JT-UGM-202538 (EPI_ISL_525492) & $\mathrm{T}$ & $P$ & $\mathrm{~L}$ & V & G & $\mathbf{H}$ & A & $\mathrm{S}$ & & \\
\hline YO-UGM-200927 (EPI_ISL_516806) & $\mathrm{T}$ & $P$ & $\mathrm{~L}$ & V & $\mathrm{D}$ & Q & A & $\mathrm{S}$ & & \\
\hline JT-UGM-47906 (EPI_ISL_576383) & $\mathrm{T}$ & $P$ & $\mathrm{~L}$ & V & G & Q & A & S & & \\
\hline JT-UGM-47964 (EPI_ISL_610162) & $\mathrm{T}$ & $P$ & $\mathrm{~L}$ & V & G & Q & A & S & & \\
\hline JT-UGM-48660 (EPI_ISL_610161) & $\mathrm{T}$ & $P$ & $\mathrm{~L}$ & A & G & $\mathrm{H}$ & $\mathrm{S}$ & I & & \\
\hline YO-UGM-00061 (EPI_ISL_575331) & $\mathrm{T}$ & $P$ & L & V & G & H & A & $\mathrm{S}$ & & \\
\hline YO-UGM-48651 (EPI_ISL_632937) & $\mathrm{T}$ & $P$ & $\mathrm{~L}$ & V & G & $\mathrm{H}$ & A & $\mathrm{S}$ & & \\
\hline YO-UGM-107727 (EPI_ISL_576145) & $\mathrm{T}$ & $P$ & $\mathrm{~L}$ & V & G & Q & A & $\mathrm{S}$ & & \\
\hline
\end{tabular}

Family clusters are indicated in asterisks: Case-1 (*) and Case-2 (**). In family cluster-1 and 2, variations in the amino acids of the NSP2 protein (V247A and T256I, and T256I and Q321K, respectively) were noted, but not in others. The virus samples isolated from fatal disease outcomes of family cluster-1 (YO-UGM-10001|EPI_ ISL_576113 and YO-UGM-10003|EPI_ISL_576115) carried V247A mutation in the NSP2 protein, while those from the recovered patient (YO-UGM-10002|EPI_

ISL_576114) did not. Bold, amino acids were different from their reference (Wuhan/19 [NC_045512.2])

the spike protein or other proteins or SNPs and severity of COVID-19.

Phylogenetic analysis revealed that three viruses from family cluster-1 formed a monophyletic group.
The epidemiological and genetic data indicated that local transmission occurred in family cluster-1 in which patient-1.1 (YO-UGM-10002|EPI_ISL_576114) was initially infected and then transmitted the virus 


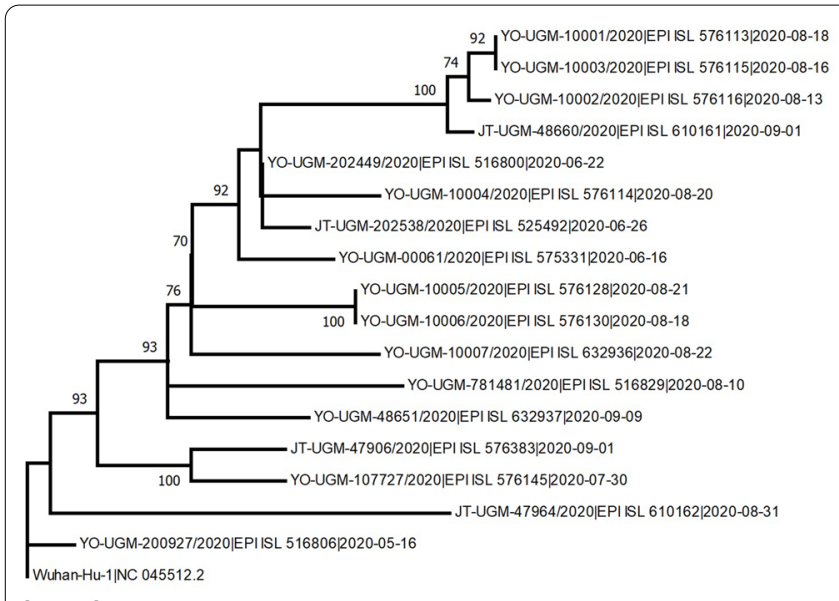

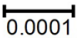

(a)

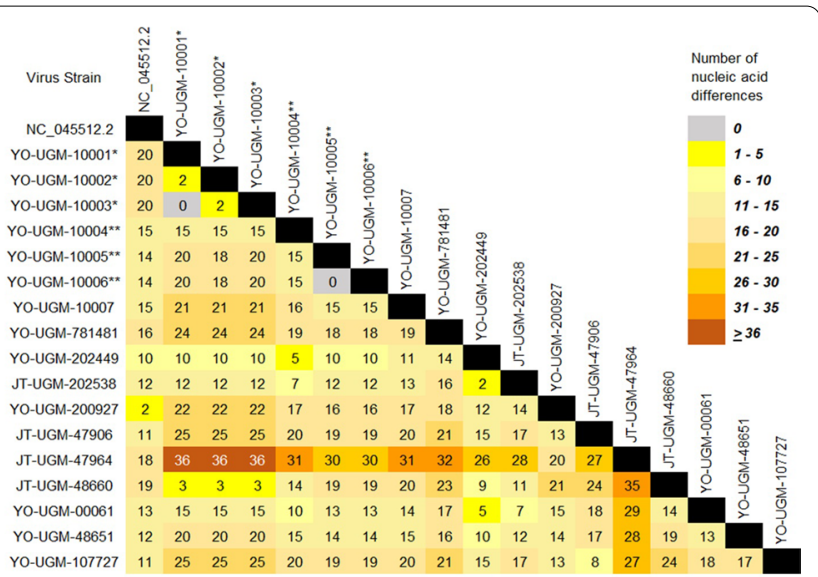

(b)

Fig. 3 Phylogenetic analysis and nucleic acid differences of family clusters' virus sequences compared to the other SARS-CoV-2 virus sequences from Yogyakarta and Central Java. a The tree was constructed from $29.409 \mathrm{nt}$ length of the open reading frame (ORF) of 142 SARS-CoV-2 virus sequences using Neighbor Joining statistical method and computed using the Kimura 2-parameter method with 2,000 bootstrap replications. The tree is rooted to Wuhan/Hu-1/2019 (NC_045512.2) with the bootstrap percentage values less than $70 \%$ hidden and it is drawn to scale (0.0001) with branch lengths measured in the number of substitutions per site. $\mathbf{b}$ The number of base differences per sequence from between sequences are shown. Codon positions included were $1 s t+2 n d+3 r d+$ Noncoding. All ambiguous positions were removed for each sequence pair. There was a total of 29,409 positions in the final dataset. Family clusters are indicated in asterisks: Case-1 (*) and Case-2 (**)

to patient-1.2 (YO-UGM-10003|EPI_ISL_576115) and patient-1.3 (YO-UGM-10001|EPI_ISL_576113). Interestingly, the virus that infected patient 2.2 in family cluster- 2 was genetically different from that which infected both two counterparts: patient 2.1 (YO-UGM-10006/2020 (EPI_ISL_576130) and patient 2.3 (YO-UGM-10005/2020 (EPI_ISL_576128). These viruses formed a polyphyletic group indicating there is the possibility of different sources of infection (two convergent descendants, but not their common ancestors).

Recently, more than $50 \%$ of the viral genome sequences in the UK were reported to have a new single phylogenetic cluster, i.e. B.1.1.7 variant (multiple spike protein mutations: deletion 69-70, deletion 144, N501Y, A570D, D614G, P681H, T716I, S982A, D1118H) [9]. These new variants have been associated with a higher transmissibility of SARS-CoV-2 up to 70\% [9]. Until the submission date of April 2021 in GISAID, these variants were also detected in Asia, including Indonesia [11]. Interestingly, we detected other spike protein mutations in our collected virus strains, including those from the family clusters, i.e. L5F, V213A, W258R, Q677H, and K811I. Noteworthy, the V213A variant was identified in all patients from family cluster-1. V213A was detected in 4/17 (23.5\%) of our samples. This variant is only found in only $0.01 \%$ of samples in four countries, including Indonesia [11]. Whether this variant is due to a founder effect needs further study.

Currently, besides the D614G variant, several mutations within the receptor binding domains (RBD) of the
$S$ protein have attracted most scientists' attention due to their increased frequency in certain countries, including S477N (Australia and some Central European), N439K (UK and European), and N501Y (part of the new UK variant B.1.1.7, the new South Africa variant 501.V2 and the new Brazil variant P.1) [11]. These variants might be associated with some potential advantages for these viruses. While the B.1.1.7 variant has been associated with COVID-19 clinical severity $[17,18]$, the 501.V2 and P.1 variants have not [20].

In addition, among eight clades in the GISAID classification, we only detected five clades, i.e. L, G, GH, $\mathrm{GR}$, and $\mathrm{O}$, in the SARS-CoV-2 samples from Indonesia and most of them $(\sim 60 \%)$ contained D614G. Globally, D614G has been detected in $\sim 97 \%$ samples in 182 countries [11]. While a recent study showed that D614G mutation is significantly associated with the increase of SARS-CoV-2 infectivity, competitive fitness, and transmission in primary human airway epithelial cells and hamsters [21], it does not associate with the clinical severity of COVID-19 patients [22]. Moreover, it is difficult to assess the convergent evolution of D614G mutation in our samples since all samples were from Yogyakarta and Central Java and D614G has been already found in most samples (97\%) from all over the world [11]. These findings were compatible with previous studies [22, 23]. The hypothesis of convergent evolution for D614G mutation is not supported by the sequence data since almost all 614G variants derived 
from the same ancestor [23]. Volz et al. [22] proposed a more complex selective landscape in the spike protein for the co-occurring variants between D614G and the neighbouring sites (615 and 613).

Phylogenetic analysis showed that the full-genome sequences of SARS-CoV-2 identified within these family clusters are identical, which strongly indicates a direct transmission within these families. Moreover, our study is also able to determine the virus clades of COVID-19 cases with unknown contact history with a confirmed COVID-19 case. Our findings support a previous suggestion regarding the importance of genomic epidemiology in filling the gaps of identifying SARS$\mathrm{CoV}-2$ infection sources [6]. Therefore, a full-genome surveillance of SARS-CoV-2 in Indonesia is essential to prevent further transmission of SARS-CoV-2 and to identify any established or new variant that might affect the SARS-CoV-2 transmission and severity.

Notably, our study only included a limited number of family clusters from Yogyakarta and Central Java, Indonesia. These limitations should be considered for interpretations of our findings.

\section{Conclusions}

This is the first molecular epidemiology study associating the full-genome sequences of SARS-CoV-2 with the epidemiological and clinical data within family clusters. Phylogenetic analysis revealed that the three viruses from family cluster-1 formed a monophyletic group, whereas viruses from family cluster- 2 formed a polyphyletic group indicating there is the possibility of different sources of infection. This study highlights how the same spike protein mutations among members of the same family might show different disease outcomes. Moreover, we also detected multiple spike protein mutations in our samples. Further studies are necessary to clarify the impact of these multiple spike protein mutations in the transmission and severity of SARS-CoV-2 infection, especially in Indonesia.

\section{Abbreviation}

SNPs: Single nucleotide polymorphisms.

\section{Supplementary Information}

The online version contains supplementary material available at https://doi. org/10.1186/s12920-021-00990-3.

Additional file 1: Table S1. Ninety-four SNPs were detected throughout the ORP of the SARS-CoV-2 virus samples with $60 \%$ (54/94) of the nucleic acid changes resulting in amino acid substitutions (missense mutations).

Additional file 2: Table S2 The Acknowledgments Table for GISAID is report.

\section{Acknowledgements}

We thank the technical support from Sri Fatmawati (Faculty of Medicine, Public Health and Nursing, Universitas Gadjah Mada), Safitriani and Muhammad Taufiq Soekarno (PT. Pandu Biosains). We also gratefully acknowledge the authors, the originating and submitting Laboratories for their sequence and metadata shared through GISAID. All submitters of data may be contacted directly via www.gisaid.org. The Acknowledgments Table for GISAID is reported as Additional file 2: Table S2. Yogyakarta-Central Java COVID-19 Study Group members: Elisabeth S. Herini ${ }^{7}$, Titis Widowati ${ }^{7}$, Cahya Dewi Satria$^{7}$, Sumardi ${ }^{5}$, Bambang Sigit Riyanto ${ }^{5}$, Munawar Gani ${ }^{5}$, Satria Maulana ${ }^{5}$, Ludhang Pradipta Rizki ${ }^{3}$, Umi Solekhah Intansari ${ }^{6}$, Elizabeth Henny Herningtiyas ${ }^{6}$, Nur Imma Fatimah Harahap ${ }^{6}$, Bagoes Poermadjaja ${ }^{2}$, Sintong HMT Hutasoit ${ }^{2}$, Indaryati ${ }^{8}$, Havid Setyawan ${ }^{8}$, Kemala Athollah ${ }^{4}$, Maria Patricia Inggriani ${ }^{4}$.

\section{Authors' contributions}

$\mathrm{G}, \mathrm{HW}, \mathrm{MSH}, \mathrm{Kl}$, and NA conceived the study. G drafted the manuscript, and $\mathrm{HW}, \mathrm{MSH}, \mathrm{RT}, \mathrm{A}, \mathrm{Kl}, \mathrm{S}, \mathrm{EA}$, and TW critically revised the manuscript for important intellectual content. G, MSH, M, IT, REK, RT, I, S, EWD, ES, DAAD, EB, HR, YP, IP, OS, DA, SS, WW, DAP, FF, UW, ARF, ASK, NRA, AS, DS, ISL, and TA collected the data; and $G, M$, and $H W$ analyzed the data. All authors read and approved the final manuscript.

\section{Funding}

Our study was funded by Indonesian Ministry of Research and Technology/ National Agency for Research and Innovation. The funders had no role in study design, data collection and analysis, decision to publish, or preparation of the manuscript.

\section{Availability of data and materials}

All data generated or analyzed during this study are included in the submission. The sequence and metadata are shared through GISAID (www.gisaid. org).

\section{Declarations}

Consent to publish

All participants or guardians signed a written informed consent for participating in this study.

\section{Competing interests}

The authors declared no potential conflicts of interest with respect to the research, authorship, and/or publication of this article.

\section{Author details}

${ }^{1}$ Pediatric Surgery Division, Department of Surgery/Genetics Working Group Faculty of Medicine, Public Health and Nursing, Universitas Gadjah Mada/Dr, Sardjito Hospital, Jl. Kesehatan No. 1, Yogyakarta 55281, Indonesia. ${ }^{2}$ Disease Investigation Center Wates, Directorate General of Livestock and Animal Health Services, Ministry of Agriculture, Yogyakarta, Indonesia. ${ }^{3}$ Department of Microbiology, Faculty of Medicine, Public Health and Nursing, Universitas Gadjah Mada, Yogyakarta, Indonesia. ${ }^{4}$ Genetics Working Group, Faculty of Medicine, Public Health and Nursing, Universitas Gadjah Mada, Yogyakarta, Indonesia. ${ }^{5}$ Pulmonology Division, Department of Internal Medicine, Faculty of Medicine, Public Health and Nursing, Universitas Gadjah Mada/Dr, Sardjito Hospital, Yogyakarta, Indonesia. ${ }^{6}$ Department of Clinical Pathology and Laboratory Medicine, Faculty of Medicine, Public Health and Nursing, Universitas Gadjah Mada/Dr, Sardjito Hospital, Yogyakarta 55281, Indonesia. ${ }^{7}$ Department of Child Health, Faculty of Medicine, Public Health and Nursing, Universitas Gadjah Mada/Dr, Sardjito Hospital, Yogyakarta, Indonesia. ${ }^{8}$ Balai Besar Teknik Kesehatan Lingkungan Dan Pengendalian Penyakit, Yogyakarta, Yogyakarta, Indonesia. ${ }^{9}$ Department of Computer Science and Electronics Faculty of Mathematics and Natural Sciences, Universitas Gadjah Mada, Yogyakarta, Indonesia. ${ }^{10}$ Department of Child Health/Genetics Working Group, Faculty of Medicine, Public Health and Nursing, Universitas Gadjah Mada/UGM Academic Hospital, Yogyakarta, Indonesia. ${ }^{11}$ Department of Physiology, Faculty of Medicine, Public Health and Nursing, Universitas Gadjah Mada/UGM Academic Hospital, Yogyakarta, Indonesia. ${ }^{12}$ Department of Anatomical Pathology/Genetics Working Group, Faculty of Medicine, Public Health and Nursing, Universitas Gadjah Mada, Yogyakarta, Indonesia. ${ }^{13}$ Department of Microbiology, Faculty of Medicine, Public Health and Nursing, Universitas Gadjah Mada, UGM 
Academic Hospital, Yogyakarta, Indonesia. ${ }^{14}$ Centre of Tropical Medicine, Faculty of Medicine, Public Health and Nursing, Universitas Gadjah Mada, Yogyakarta, Indonesia. ${ }^{15}$ Department of Pharmacology and Therapy/Genetics Working Group, Faculty of Medicine, Public Health and Nursing, Universitas Gadjah Mada, Yogyakarta, Indonesia.

Received: 9 February 2021 Accepted: 18 May 2021

Published online: 01 June 2021

\section{References}

1. World Health Organization. https://www.who.int/news-room/detail/2704-2020-who-timeline---covid-19. Accessed 9 Feb 2021.

2. Phelan AL, Katz R, Gostin LO. The novel coronavirus originating in Wuhan, China: challenges for global health governance. JAMA. 2020;323:709-10. https://doi.org/10.1001/jama.2020.1097.

3. World Health Organization. https://covid19.who.int/table. Accessed 9 Feb 2021.

4. Liu T, Gong D, Xiao J, Hu J, He G, Rong Z, Ma W. Cluster infections play important roles in the rapid evolution of COVID-19 transmission: a systematic review. Int J Infect Dis. 2020;99:374-80.

5. Zhang H, Hong C, Zheng Q, Zhou P, Zhu Y, Zhang Z, Bi Q, Ma T. A multi-family cluster of COVID-19 associated with asymptomatic and presymptomatic transmission in Jixi City, Heilongjiang, China, 2020. Emerg Microbes Infect. 2020;9:2509-14.

6. Pattabiraman C, Habib F, Harsha PK, Rasheed R, Prasad P, Reddy V, Dinesh P, Damodar T, Hosallimath K, George AK, Kiran Reddy NV, John B, Pattanaik A, Kumar N, Mani RS, Venkataswamy MM, Shahul Hameed SK, Kumar BGP, Desai A, Vasanthapuram R. Genomic epidemiology reveals multiple introductions and spread of SARS-CoV-2 in the Indian state of Karnataka. PLOS ONE. 2020;15:e0243412.

7. Lu J, du Plessis L, Liu Z, Hill V, Kang M, Lin H, Sun J, François S, Kraemer MUG, Faria NR, McCrone JT, Peng J, Xiong Q, Yuan R, Zeng L, Zhou P, Liang C, Yi L, Liu J, Xiao J, Hu J, Liu T, Ma W, Li W, Su J, Zheng H, Peng B, Fang S, Su W, Li K, Sun R, Bai R, Tang X, Liang M, Quick J, Song T, Rambaut A, Loman N, Raghwani J, Pybus OG, Ke C. Genomic epidemiology of SARSCoV-2 in Guangdong Province. China Cell. 2020;181:997-1003.e9.

8. MacLean OA, Orton RJ, Singer JB, Robertson DL. No evidence for distinct types in the evolution of SARS-CoV-2. Virus Evol. 2020;6:veaa034.

9. European Centre for Disease Prevention and Control. Rapid increase of a SARS-CoV-2 variant with multiple spike protein mutations observed in the United Kingdom—20 December 2020. Stockholm: ECDC; 2020.

10. About UGENE - Unipro UGENE Online User Manual v. 1.30 - WIKI [Internet]. Ugene.net. 2020 [cited 22 December 2020]. https://ugene.net/wiki/ display/UUOUM30/About+UGENE.
11. GISAID. 2020. Pandemic coronavirus causing COVID-19. https://platform. gisaid.org/epi3/cfrontend\#8dc5e. Accessed 21 Dec 2020.

12. Gunadi WH, Marcellus HMS, Daniwijaya EW, Rizki LP, Supriyati E, Nugrahaningsih DAA, Afiahayati S, Iskandar K, Anggorowati N, Kalim AS, Puspitarani DA, Athollah K, Arguni E, Nuryastuti T, Wibawa T. Fulllength genome characterization and phylogenetic analysis of SARS-CoV-2 virus strains from Yogyakarta and Central Java. Indonesia PeerJ. 2020;8:e10575. https://doi.org/10.7717/peerj.10575.

13. Xia X. DAMBE7: New and improved tools for data analysis in molecular biology and evolution. Mol Biol Evol. 2018;35:1550-2.

14. Kumar S, Stecher G, Li M, Knyaz C, Tamura K. MEGA X: Molecular Evolutionary Genetics Analysis across computing platforms. Mol Biol Evol. 2018:35:1547-9.

15. Beeching NJ, Fletcher TE, Fowler R. BMJ best practice. Coronavirus Disease 2019 (COVID-19). https://bestpractice.bmj.com/topics/en-us/3000168/ prognosis. Accessed 10 Oct 2020.

16. Mission J. Report of the WHO-China joint mission on Coronavirus Disease 2019 (COVID-19). WHO; 2020.

17. Davies NG, Jarvis Cl, CMMID COVID-19 Working Group, Edmunds WJ, Jewell NP, Diaz-Ordaz K, Keogh RH. Increased mortality in communitytested cases of SARS-CoV-2 lineage B.1.1.7. Nature. 2021. https://doi.org/ 10.1038/s41586-021-03426-1.

18. Horby P, Huntley C, Davies N, et al. NERVTAG note on B.1.1.7 severity. SAGE meeting report. January 21, 2021.

19. Severe Covid-19 GWAS Group, Ellinghaus D, Degenhardt F, Bujanda L, Buti M, Albillos A, et al. Genomewide association study of severe Covid-19 with respiratory failure. N Engl J Med. 2020;383(16):1522-34.

20. CDC. Science Brief: Emerging SARS-CoV-2 Variants https://www.cdc.gov/ coronavirus/2019-ncov/science/science-briefs/scientific-brief-emergingvariants.htm|\#ref2. Accessed 14 Apr 2021.

21. Hou YJ, Chiba S, Halfmann P, Ehre C, Kuroda M, Dinnon KH 3rd, et al. SARSCoV-2 D614G variant exhibits efficient replication ex vivo and transmission in vivo. Science. 2020;370:1464-8.

22. Volz E, Hill V, McCrone JT, Price A, Jorgensen D, O'Toole Á, et al. Evaluating the effects of SARS-CoV-2 spike mutation D614G on transmissibility and pathogenicity. Cell. 2021;184(1):64-75.e11.

23. van Dorp L, Richard D, Tan CCS, Shaw LP, Acman M, Balloux F. No evidence for increased transmissibility from recurrent mutations in SARSCoV-2. Nat Commun. 2020;11:5986.

\section{Publisher's Note}

Springer Nature remains neutral with regard to jurisdictional claims in published maps and institutional affiliations.
Ready to submit your research? Choose BMC and benefit from:

- fast, convenient online submission

- thorough peer review by experienced researchers in your field

- rapid publication on acceptance

- support for research data, including large and complex data types

- gold Open Access which fosters wider collaboration and increased citations

- maximum visibility for your research: over $100 \mathrm{M}$ website views per year

At BMC, research is always in progress.

Learn more biomedcentral.com/submissions 